Research Paper

\title{
The Association of Mitofusion-2 Gene Polymorphisms with Susceptibility of Essential Hypertension in Northern Han Chinese Population
}

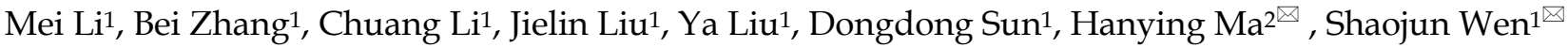 \\ 1. Department of Hypertension Research, Beijing Anzhen Hospital, Capital Medical University and Beijing Institute of Heart Lung and Blood Vessel Diseases, \\ Beijing, People's Republic of China \\ 2. Department of Cardiology, Beijing Anzhen Hospital, Capital Medical University, Beijing, People's Republic of China
}

$\square$ Corresponding authors: Shaojun Wen, Department of Hypertension Research, Beijing Anzhen Hospital, Capital Medical University and Beijing Institute of Heart Lung and Blood vessel Diseases, 2 Anzhen Road, Chaoyang District, Beijing 100029, PR China. Tel: +86-10-64456268; Fax: +86-10-64416527; E-mail: wenshaojun@ccmu.edu.cn. Hanying Ma, Department of Cardiology, Beijing Anzhen Hospital, Capital Medical University, 2 Anzhen Road, Chaoyang District, Beijing 100029, PR China. Tel: +86-10-64456416; E-mail: mahanying@126.com

( ) Ivyspring International Publisher. Reproduction is permitted for personal, noncommercial use, provided that the article is in whole, unmodified, and properly cited. See http://ivyspring.com/terms for terms and conditions.

Received: 2015.06.22; Accepted: 2015.11.26; Published: 2016.01.01

\begin{abstract}
Background: Mitofusion-2 (Mfn2) played an important role in regulating vascular smooth muscle cells proliferation, insulin resistance and endoplasmic reticulum stress, which were found to be involved in the development of hypertension. So we inferred that the $M f n 2$ gene may participate in the pathogenesis of hypertension. The aim of this study was to determine whether common single nucleotide polymorphisms (SNPs) in Mfn2 gene were associated with essential hypertension (EH) in northern Han Chinese.

Methods: We genotyped 6 tagging SNPs of Mfn2 gene (rs2336384, rs2295281, rs17037564, rs2236057, rs2236058 and rs3766741) with the TaqMan assay in 626 hypertensive patients and 618 controls.

Results: Logistic regression analysis indicated that CC+CA genotype of rs2336384 and AA+AG genotype of $r s 2236057$ were significantly associated with increased risk of $E H(O R=1.617, P=0.005$; $\mathrm{OR}=1.418, \mathrm{P}=0.031$, respectively). $\mathrm{GG}$ genotype of $\mathrm{rs} 2236058$ and $\mathrm{GG}+\mathrm{CG}$ genotype of rs3766741 were found to be significantly associated with decreased risk of $E H(O R=0.662, P=0.023 ; O R=0.639$, $\mathrm{P}=0.024)$. When stratified by gender, for rs2336384, rs2236057 and rs2236058, significant association was observed in males, but not in females. Haplotype analysis indicated that the CCAACC haplotype was positively correlated with $\mathrm{EH}$ and there was a negative correlation between ACAGGG haplotype and $\mathrm{EH}$.

Conclusions: This study demonstrated that $M f n 2$ gene polymorphisms were associated with essential hypertension in northern Han Chinese population, especially in male subjects.
\end{abstract}

Key words: essential hypertension, mitofusion-2, polymorphism, haplotype, northern Han Chinese population

\section{Introduction}

Hypertension is a major global public health problem due to its high prevalence and its association with morbidity and mortality from stroke, myocardial infarction, congestive heart failures and end-stage renal diseases [1]. In China, it was reported that $27.2 \%$ of the adults aged 35-74 years suffered from hypertension [2].

Essential hypertension (EH) is a multifactorial disorder resulting from a complex interplay of genetic factors and environmental determinants. Approximately $20-60 \%$ of the blood pressure variation is genetically determined [3]. To date, there have been many studies searching for the hypertension-susceptibility loci. In recent years, genome-wide association studies (GWAS) have been a relatively new method in identifying the susceptibility genes of $\mathrm{EH}$. However, the findings from GWAS explain only a small fraction of genetic variants [4-6]. Considering 
the ethnic differences and the influence of environmental factors, candidate gene association study still plays an important role in exploring the potential susceptibility genes.

The gene Mfn2 (Mitofusion-2, also named Hyperplasia suppressive gene, HSG) was initially isolated using differential display technology and its expression was reduced in vascular smooth muscle cells (VSMCs) of the spontaneously hypertensive rat (SHR) [7], which suggested that the Mfn2 gene may be a hypertension-related gene. This gene is mapped to chromosome 1p36.22. Recent experimental data indicated that Mfn2 can regulate the proliferation of VSMCs [8, 9], insulin resistance [10] and endoplasmic reticulum (ER) stress [11]. Given that VSMCs proliferation, insulin resistance and ER stress are strongly associated with hypertension [12-15], so we inferred that Mfn2 gene may be involved in the development of $\mathrm{EH}$ through these pathological processes.

There were few studies investigating the relationship between the gene $M f n 2$ and $\mathrm{EH}$. Jin et al. [16] only identified 1 SNP in the Mfn2 gene and showed no significant association between rs2336384 polymorphism with hypertension in Koreans. However, Wang et al. [17] found that several polymorphisms including rs2336384 in intron 2 of $M f n 2$ gene were associated with $\mathrm{EH}$ in Chinese. Considering that Wang et al.'s study did not include all common polymorphisms in Mfn2 gene and the inconsistent association results between Chinese and Koreans, we performed another study to confirm the relationship between $\mathrm{Mfn} 2$ polymorphisms and $\mathrm{EH}$ by choosing tagging SNPs that could cover most of common polymorphisms in Mfn2 gene. The aim of the present study was to investigate associations between the $M f n 2$ gene and the risk of essential hypertension in northern Han Chinese.

\section{Materials and methods}

\section{Subjects}

All individuals were northern Han Chinese ancestry with no intermarriage. All the participants in this study were randomly recruited from the physical examination center of Beijing Anzhen Hospital of Capital Medical University, Beijing, China and another two examination centers at local health stations, Liuliqiao and Guozhuang, in Beijing suburbs. All subjects completed a standard questionnaire on personal medical history and family history of hypertension.

The blood pressure (BP) measurements were taken with a mercury sphygmomanometer by the experienced internists. Prior to BP measurements, all participants were asked to avoid cigarettes, alcohol, tea, coffee or exercise for at least 30 minutes. After the subjects had been seated on a chair with their feet on the floor and their arms supported at heart level for 10 minutes, three measurements were taken at least 5 minutes intervals. All readings were obtained from the right arm and the average of the three measurements was used for analysis.

Hypertension was defined as the average systolic blood pressure (SBP) $\geq 140 \mathrm{mmHg}$ and/or the average diastolic blood pressure (DBP) $\geq 90 \mathrm{mmHg}$ and/or self-reported current treatment for hypertension with antihypertensive medication. The control subjects had $\mathrm{SBP}<130 \mathrm{mmHg}$ and $\mathrm{DBP}<80 \mathrm{mmHg}$, respectively, and no history of antihypertensive medication. Subjects with secondary hypertension, primary renal disease, diabetes mellitus, hepatic disorders, cancers or endocrine diseases were excluded. Physical examination and serum biochemical profiles were administered to each of the participants. Information on smoking and drinking habits were obtained from the interview. Smokers were defined as cigarette consumers who had smoked no less than 100 cigarettes; Drinkers were defined as alcohol consumers who drank no less than 12 times during the year $[18,19]$. This study complied with the Declaration of Helsinki. All participants involved gave their written informed consent for the genetic analysis and the study was approved by the Ethics Committee of Beijing Anzhen Hospital of Capital Medical University, Beijing, China.

\section{SNP identification and genotyping}

The Mfn2 common SNPs (minor allele frequency $[\mathrm{MAF}]>10 \%$ ) were searched from the Han Chinese data sets of the International HapMap Project SNP database (http://www.hapmap.org/, HapMap Genome Browser release \#27). The sets tag SNPs was selected to predict the remaining common SNPs with a $\mathrm{r}^{2} \geq 0.85$ using Haploview 4.2 software (http://www.broad.mit.edu/mpg/haploview). According to the criteria, six tag SNPs (rs2336384, rs2295281, rs17037564, rs2236057, rs2236058 and rs3766741) of the Mfn2 gene were selected. Rs2336384 is located in intron 2; rs2295281 and rs17037564 are located in intron 8; rs2236057 and rs2236058 are located in intron 11; rs3766741 is located in intron 18.

Blood samples were collected using ethylenediamine tetra-acetic acid (EDTA)-anticoagulated vacutainer tubes from all subjects. Genomic DNA was extracted from the peripheral blood leukocytes with a standard phenol-chloroform method, and stored at $-80^{\circ} \mathrm{C}$. All selected SNPs were genotyped in all 1244 subjects using the TaqMan assay. Primers and probes of rs2336384 C_11461995_10, rs2295281 C_16189654_10, rs17037564 C_32800152_10, 
rs2236057C_15953633_10, rs2236058C_15953634_10, and rs3766741C_25606040_10 were obtained from Applied Biosystems Assay-by-Design Service for SNP genotyping. Genotyping reactions contained TaqMan ${ }^{\circledR}$ PCR Master Mix, No AmpErase ${ }^{\circledR}$ UNG, and about 5 ng of genomic DNA in a final volume of $5 \mu \mathrm{L}$. The GeneAmp PCR System 9700 thermal cycler (Applied Biosystems, 850 Lincoln Center Drive, Foster City, CA 94404 USA) was used for amplification. The cycling conditions were as follows: initial denaturation and activation $95^{\circ} \mathrm{C}$ for $10 \mathrm{~min}$, followed by 35 cycles of $95^{\circ} \mathrm{C}$ for $20 \mathrm{sec}$ and $62^{\circ} \mathrm{C}$ for $1 \mathrm{~min}$. Each 384-well plate contained 380 samples of an unknown genotype, two samples with no DNA but with reagents (negative control), and two duplicate samples (control). Plates were read on the ABI HT 7900 instrument using the end-point analysis mode of the SDS, version 2.0, software package (Applied Biosystems). Genotypes were discriminated by analyzing the dye-component fluorescent emission data depicted in the X-Y scatter plot of SDS software. Genotyping was performed blindly to all other data.

\section{Statistical analyses}

The SPSS (Version 17.0; SPSS, Chicago, IL, USA) software was used to carry out database management and statistical analyses. Normally continuous variables were presented as mean \pm standard deviation (SD), medians (25th/75th quartiles) were used for non-normally distributed variables and categorical variables were expressed as percentages. Comparisons between groups were done with Student's t-test, Mann-Whitney $U$ test and chi-squared test, respectively. All statistical tests were two-tailed, and $\mathrm{P}<0.05$ was defined to be statistically significant. Hardy-Weinberg equilibrium (HWE) was assessed by the chi-square for goodness of fit based on a web program (http://ihg.gsf.de/cgi-bin/hw/hwa1.pl) [20]. The genotypic and allelic frequency between cases and controls were compared by using the chi-square test. To test whether there is an association between each SNP and hypertension risk, Logistic regression was used to study the effect of the six SNPs of Mfn2 gene on hypertension status under different genetic models (additive, dominant and recessive models) after adjusting for the confounding factors. Odds ratio (ORs) and their $95 \%$ confidence interval $(95 \% \mathrm{CI})$ were calculated. Construction of the linkage disequilibrium map and haplotype blocks within polymorphisms of the $M f n 2$ gene was based on genotypes using Haploview software (version 4.2) (http://www.broad.mit.edu/mpg/haploview/). Considering the effect of the covariates on the association analysis, the haplotype-based logistic regression analysis was conducted using the PLINK software
(Version 2.0, http:// pngu.mgh.harvard.edu/ $\sim$ purcell/plink/) [21].

\section{Results}

\section{Characteristics of the subjects}

A total of 1244 unrelated participants comprising 626 hypertensive cases (411 men and 215 women; mean age $50.22 \pm 7.18$ ) and 618 normotensive controls

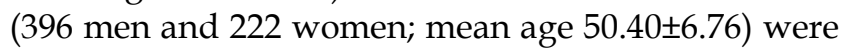
recruited for the present study. The clinical and laboratory characteristics of cases and controls were summarized in Table 1 . The subjects were adequately matched for age and gender for hypertensive cases and controls. For total subjects, males and females, when compared with the control subjects, the following variables were significantly higher in hypertensive patients: SBP, DBP, body mass index (BMI), total cholesterol (TCHO), triglyceride (TG) and glucose levels. The incidence of drinking was found to be significantly higher in the total and male hypertensive cases as compared to the control subjects. No significant differences were found for the following values between the hypertensive patients and the control subjects: age, heart rate (HR), Creatinine and low-density lipoprotein cholesterol (LDL-C). The plasma concentration of the high-density lipoprotein cholesterol (HDL-C) was found to be significantly higher in the male control groups as compared to the male hypertensive cases.

\section{Detection and distribution of the SNPs}

Among all the participants, $98.3 \%$ samples of rs2336384, 98.8\% samples of rs2295281, 99.3\% samples of rs17037564, 98.6\% samples of rs2236057, 98.9\% samples of rs2236058 and $99.3 \%$ samples of rs3766741 were successfully genotyped. The genotype frequencies for each of the six SNPs were in agreement with Hardy-Weinberg equilibrium in the total control group, in the male control group, as well as in the female group $(\mathrm{P}>0.05)$. Table 2 shows the distribution of genotype and allele frequencies for the six polymorphisms in the $M f n 2$ gene.

Chi-square analyses showed that the genotype and allele distribution of rs2336384, rs2236058 and rs3766741 differed significantly between the hypertensive cases and normotensive controls $(\mathrm{P}<0.05)$. The $\mathrm{C}$ allele of rs2336384 was significantly more prevalent in the hypertensive cases, whereas the $G$ allele frequency of rs2236058 and rs3766741 was significantly higher in the control subjects. When the subjects were subdivided by gender, similar findings for rs 2336384 and rs2236058 polymorphisms were observed in males, but not in females. For rs2236057, there was significant difference in the genotype and allele frequencies between the male hypertensive cases and 
controls. Furthermore, rs2295281 showed a significant difference in allele frequency $(\mathrm{P}<0.05)$ in males. For rs17037564, there was no significant difference in the proportion of genotypes and alleles between the two groups whether in total subjects, in females or in males.

Table 1. Characteristics of Normotensive Controls and Hypertensive Cases.

\begin{tabular}{|c|c|c|c|c|c|c|c|c|c|}
\hline Variables & Total NT(n=618) & Total EH(n=626) & $P$ value & Male NT(n=396) & Male $\mathrm{EH}(\mathrm{n}=411)$ & $P$ value & Female NT(n=222) & Female EH(n=215) & $P$ value \\
\hline Age(years) & $50.22 \pm 7.18$ & $50.40 \pm 6.76$ & 0.655 & $49.67 \pm 7.87$ & $49.68 \pm 9.16$ & 0.982 & $51.21 \pm 5.63$ & $51.80 \pm 5.66$ & 0.278 \\
\hline SBP (mmHg) & $116.94 \pm 11.79$ & $139.45 \pm 16.97$ & $<0.001$ & $116.54 \pm 10.75$ & $139.26 \pm 16.70$ & $<0.001$ & $117.65 \pm 13.47$ & $139.80 \pm 17.52$ & $<0.001$ \\
\hline DBP (mmHg) & $76.67 \pm 8.51$ & $91.88 \pm 12.11$ & $<0.001$ & $77.03 \pm 8.30$ & $94.18 \pm 11.95$ & $<0.001$ & $76.02 \pm 8.85$ & $87.48 \pm 11.17$ & $<0.001$ \\
\hline $\operatorname{BMI}\left(\mathrm{kg} / \mathrm{m}^{2}\right)$ & $24.99 \pm 3.20$ & $27.01 \pm 3.39$ & $<0.001$ & $25.11 \pm 3.12$ & $27.48 \pm 3.23$ & $<0.001$ & $24.77 \pm 3.34$ & $26.10 \pm 3.53$ & $<0.001$ \\
\hline HR(bpm) & $71.64 \pm 9.51$ & $71.47 \pm 9.81$ & 0.798 & $70.98 \pm 10.22$ & $71.25 \pm 9.97$ & 0.766 & $72.48 \pm 8.47$ & $71.87 \pm 9.52$ & 0.540 \\
\hline Creatinine(mmol/L) & $77.07 \pm 14.14$ & $78.63 \pm 18.82$ & 0.133 & $82.42 \pm 14.02$ & $84.02 \pm 18.93$ & 0.244 & $68.18 \pm 8.90$ & $68.44 \pm 13.74$ & 0.830 \\
\hline TCHO (mmol/L) & $5.01 \pm 0.90$ & $5.32 \pm 1.65$ & $<0.001$ & $5.03 \pm 0.92$ & $5.24 \pm 1.59$ & 0.023 & $4.98 \pm 0.88$ & $5.49 \pm 1.75$ & $<0.001$ \\
\hline TG (mmol/L) & $1.35(0.95-1.99)$ & $1.85(1.27-2.56)$ & $<0.001$ & $1.49(1.04-2.20)$ & $1.96(1.31-2.79)$ & $<0.001$ & $1.09(0.83-1.58)$ & $1.63(1.18-2.16)$ & $<0.001$ \\
\hline LDL-C (mmol/L) & $3.45 \pm 0.77$ & $3.37 \pm 0.87$ & 0.208 & $3.46 \pm 0.79$ & $3.33 \pm 0.86$ & 0.089 & $3.37 \pm 0.71$ & $3.46 \pm 0.94$ & 0.820 \\
\hline HDL-C(mmol/L) & $1.27 \pm 0.31$ & $1.20 \pm 0.60$ & 0.081 & $1.22 \pm 0.30$ & $1.12 \pm 0.64$ & 0.045 & $1.44 \pm 0.26$ & $1.34 \pm 0.50$ & 0.182 \\
\hline Glucose(mmol/L) & $4.99 \pm 0.59$ & $5.36 \pm 0.61$ & $<0.001$ & $5.04 \pm 0.62$ & $5.38 \pm 0.61$ & $<0.001$ & $4.89 \pm 0.53$ & $5.30 \pm 0.59$ & $<0.001$ \\
\hline Smokers(\%) & $114(28.1)$ & 173(28.9) & 0.782 & $105(39.2)$ & $166(41.7)$ & 0.515 & $9(6.5)$ & $7(3.5)$ & 0.195 \\
\hline Drinkers(\%) & $65(15.9)$ & $193(32.2)$ & $<0.001$ & $56(20.8)$ & $179(45.1)$ & $<0.001$ & $9(6.4)$ & $14(6.9)$ & 0.842 \\
\hline
\end{tabular}

Continuous variables were expressed as means \pm standard deviations when normally distributed and as median (interquartile range) when asymmetrically distributed.

BMI, body mass index; DBP, diastolic blood pressure; EH, essential hypertensive patients; HDL-C, high-density lipoprotein; HR, heart rate; LDL-C, low-density lipoprotein; NT, normotensive subjects; SBP, systolic blood pressure; TCHO, total cholesterol; TG, triglyceride.

Table 2. Genotype Distribution and Allele Frequency of Mfn2 Gene in Case and Control Group

\begin{tabular}{|c|c|c|c|c|c|c|c|c|c|}
\hline \multirow[t]{2}{*}{ SNP } & & & \multicolumn{3}{|c|}{ Genotype (frequency, \%) } & \multirow[t]{2}{*}{ P value } & \multicolumn{2}{|c|}{ Allele (frequency, \%) } & \multirow[t]{2}{*}{ P value } \\
\hline & & & $\mathrm{CC}$ & $\mathrm{CA}$ & AA & & $\mathrm{C}$ & A & \\
\hline \multirow[t]{7}{*}{ rs2336384 } & Total & case & $142(23.0)$ & $330(53.5)$ & $145(23.5)$ & & $614(49.8)$ & $620(50.2)$ & \\
\hline & & control & $124(20.5)$ & $297(49.0)$ & $185(30.5)$ & 0.021 & $545(45.0)$ & $667(55.0)$ & 0.018 \\
\hline & Male & case & $94(23.2)$ & $219(54.1)$ & $92(22.7)$ & & $407(50.2)$ & $403(49.8)$ & \\
\hline & & control & 75 (19.2) & $189(48.5)$ & $126(32.3)$ & 0.009 & $339(43.5)$ & $441(56.5)$ & 0.007 \\
\hline & Female & case & $48(22.6)$ & $111(52.4)$ & $53(25.0)$ & & $207(48.8)$ & $217(51.2)$ & \\
\hline & & control & $49(22.7)$ & $108(50.0)$ & $59(27.3)$ & 0.846 & $206(47.7)$ & $226(52.3)$ & 0.74 \\
\hline & & & TT & CT & $\mathrm{CC}$ & & $\mathrm{T}$ & $\mathrm{C}$ & \\
\hline \multirow[t]{7}{*}{ rs2295281 } & Total & case & $77(12.4)$ & $300(48.3)$ & $244(39.3)$ & & $454(36.6)$ & $788(63.4)$ & \\
\hline & & control & 95 (15.6) & $288(47.4)$ & $225(37.0)$ & 0.251 & 478 (39.3) & $738(60.7)$ & 0.159 \\
\hline & Male & case & $50(12.3)$ & $194(47.5)$ & $164(40.2)$ & & $294(36.0)$ & $522(64.0)$ & \\
\hline & & control & 68 (17.5) & $184(47.3)$ & $137(35.2)$ & 0.083 & $320(41.1)$ & $458(58.9)$ & 0.036 \\
\hline & Female & case & 27 (12.7) & $106(49.8)$ & $80(37.6)$ & & $160(37.6)$ & $266(62.4)$ & \\
\hline & & control & $27(12.3)$ & $104(47.5)$ & $88(40.2)$ & 0.854 & $158(36.1)$ & $280(63.9)$ & 0.651 \\
\hline & & & GG & AG & $\mathrm{AA}$ & & G & A & \\
\hline \multirow[t]{7}{*}{ rs17037564 } & Total & case & $9(1.4)$ & 117 (18.8) & 495 (79.7) & & 135 (10.9) & 1107 (89.1) & \\
\hline & & control & 7 (1.1) & 102 (16.6) & 505 (82.2) & 0.492 & $116(9.4)$ & $1112(90.6)$ & 0.242 \\
\hline & Male & case & $5(1.2)$ & 78 (19.1) & 325 (79.7) & & $88(10.8)$ & $728(89.2)$ & \\
\hline & & control & $4(1.0)$ & 68 (17.3) & $321(81.7)$ & 0.779 & $76(9.7)$ & $710(90.3)$ & 0.462 \\
\hline & Female & case & $4(1.9)$ & 39 (18.3) & $170(79.8)$ & & 47 (11.0) & $379(89.0)$ & \\
\hline & & control & $3(1.4)$ & $34(15.4)$ & $184(83.3)$ & 0.672 & $40(9.0)$ & $402(91.0)$ & 0.331 \\
\hline & & & AA & AG & GG & & A & G & \\
\hline \multirow[t]{7}{*}{ rs2236057 } & Total & case & 125 (20.3) & $301(48.8)$ & $191(31.0)$ & & $551(44.7)$ & $683(55.3)$ & \\
\hline & & control & 105 (17.2) & $292(47.9)$ & $213(34.9)$ & 0.219 & $502(41.1)$ & 718 (58.9) & 0.08 \\
\hline & Male & case & $86(21.2)$ & $193(47.7)$ & $126(31.1)$ & & $365(45.1)$ & 445 (54.9) & \\
\hline & & control & 59 (15.1) & $187(47.9)$ & $144(36.9)$ & 0.049 & 305 (39.1) & $475(60.9)$ & 0.016 \\
\hline & Female & case & 39 (18.4) & 108 (50.9) & $65(30.7)$ & & $186(43.9)$ & $238(56.1)$ & \\
\hline & & control & $46(20.9)$ & 105 (47.7) & 69 (31.4) & 0.744 & $197(44.8)$ & $243(55.2)$ & 0.789 \\
\hline & & & GG & CG & CC & & G & C & \\
\hline \multirow[t]{7}{*}{ rs2236058 } & Total & case & 120 (19.4) & 307 (49.8) & $190(30.8)$ & & $547(44.3)$ & $687(55.7)$ & \\
\hline & & control & $155(25.3)$ & $293(47.8)$ & 165 (26.9) & 0.038 & $603(49.2)$ & $623(50.8)$ & 0.016 \\
\hline & Male & case & $77(19.0)$ & 201 (49.5) & $128(31.5)$ & & 355 (43.7) & $457(56.3)$ & \\
\hline & & control & 109 (27.7) & $183(46.6)$ & $101(25.7)$ & 0.009 & $401(51.0)$ & $385(49.0)$ & 0.003 \\
\hline & Female & case & $43(20.4)$ & $106(50.2)$ & $62(29.4)$ & & $192(45.5)$ & $230(54.5)$ & \\
\hline & & control & $46(20.9)$ & $110(50.0)$ & $64(29.1)$ & 0.991 & $202(45.9)$ & $238(54.1)$ & 0.904 \\
\hline & & & GG & CG & $\mathrm{CC}$ & & G & C & \\
\hline \multirow[t]{6}{*}{ rs3766741 } & Total & case & $0(0)$ & $93(15.0)$ & $528(85.0)$ & & $93(7.5)$ & 1149 (92.5) & \\
\hline & & control & $4(0.7)$ & $112(18.2)$ & 498 (81.1) & 0.029 & $120(9.8)$ & $1108(90.2)$ & 0.043 \\
\hline & Male & case & $0(0)$ & $62(15.2)$ & $346(84.8)$ & & $62(7.6)$ & 754 (92.4) & \\
\hline & & control & $4(1.0)$ & 69 (17.5) & $321(81.5)$ & 0.08 & 77 (9.8) & $711(90.2)$ & 0.122 \\
\hline & Female & case & $0(0)$ & 31 (14.6) & $182(85.4)$ & & $31(7.3)$ & 395 (92.7) & \\
\hline & & control & $0(0)$ & 43 (19.5) & $177(80.5)$ & 0.202 & $43(9.8)$ & 397 (90.2) & 0.189 \\
\hline
\end{tabular}


Table 3. Association of Mfn2 Gene Polymorphisms with Essential Hypertension under Different Genetic Models

\begin{tabular}{|c|c|c|c|c|c|c|c|c|}
\hline \multirow[t]{2}{*}{ SNP } & \multirow[t]{2}{*}{ Models } & \multirow[t]{2}{*}{ Genotype } & \multicolumn{2}{|l|}{ Overall } & \multicolumn{2}{|l|}{ Male } & \multicolumn{2}{|l|}{ Female } \\
\hline & & & $\mathrm{OR}(95 \% \mathrm{CI})^{\mathrm{a}}$ & $\mathrm{Pa}$ & $\mathrm{OR}(95 \% \mathrm{CI})^{\mathrm{b}}$ & $\mathrm{Pb}$ & $\mathrm{OR}(95 \% \mathrm{CI})^{\mathrm{c}}$ & Pc \\
\hline \multirow[t]{3}{*}{ rs2336384 } & additive & CC vs. CA vs. AA & $1.273(1.023-1.584)$ & 0.031 & 1.407(1.005-1.97) & 0.047 & $1.160(0.798-1.687)$ & 0.437 \\
\hline & dominant & $(\mathrm{CC}+\mathrm{CA})$ vs. AA & $1.617(1.155-2.264)$ & 0.005 & $1.89(1.163-3.071)$ & 0.01 & $1.373(0.754-2.500)$ & 0.300 \\
\hline & recessive & $\mathrm{CC}$ vs. $(\mathrm{CA}+\mathrm{AA})$ & $1.120(0.773-1.622)$ & 0.549 & $1.124(0.620-2.038)$ & 0.7 & $1.071(0.579-1.980)$ & 0.828 \\
\hline \multirow[t]{3}{*}{ rs2295281 } & additive & TT vs. CT vs. CC & $0.929(0.746-1.156)$ & 0.509 & $0.881(0.644-1.206)$ & 0.428 & $1.002(0.683-1.472)$ & 0.990 \\
\hline & dominant & $(\mathrm{TT}+\mathrm{CT})$ vs. $\mathrm{CC}$ & $1.032(0.759-1.404)$ & 0.841 & $0.953(0.604-1.505)$ & 0.837 & $1.212(0.717-2.049)$ & 0.473 \\
\hline & recessive & TT vs. $(\mathrm{CT}+\mathrm{CC})$ & $0.711(0.465-1.089)$ & 0.117 & $0.697(0.389-1.251)$ & 0.226 & $0.657(0.299-1.441)$ & 0.294 \\
\hline \multirow[t]{3}{*}{ rs17037564 } & additive & GG vs. AG vs. AA & $1.035(0.737-1.454)$ & 0.841 & $0.983(0.605-1.597)$ & 0.946 & $1.253(0.702-2.235)$ & 0.446 \\
\hline & dominant & $(\mathrm{GG}+\mathrm{AG})$ vs. AA & $1.055(0.724-1.538)$ & 0.779 & $0.982(0.57-1.69)$ & 0.947 & $1.341(0.694-2.591)$ & 0.383 \\
\hline & recessive & GG vs. (AG+AA) & $0.881(0.250-3.109)$ & 0.844 & $0.974(0.17-5.576)$ & 0.976 & $0.998(0.149-6.698)$ & 0.998 \\
\hline \multirow[t]{3}{*}{ rs2236057 } & additive & AA vs. AG vs. GG & $1.234(0.997-1.527)$ & 0.053 & $1.341(0.968-1.856)$ & 0.077 & $1.057(0.736-1.517)$ & 0.765 \\
\hline & dominant & $(\mathrm{AA}+\mathrm{AG})$ vs. $\mathrm{GG}$ & $1.418(1.033-1.948)$ & 0.031 & $1.665(1.052-2.635)$ & 0.03 & $1.177(0.670-2.069)$ & 0.570 \\
\hline & recessive & AA vs. (AG+GG) & $1.192(0.810-1.754)$ & 0.373 & $1.149(0.623-2.121)$ & 0.657 & $0.964(0.513-1.810)$ & 0.910 \\
\hline \multirow[t]{3}{*}{ rs2236058 } & additive & GG vs. CG vs. CC & $0.802(0.649-0.991)$ & 0.041 & $0.746(0.542-1.027)$ & 0.072 & $0.892(0.621-1.282)$ & 0.537 \\
\hline & dominant & $(\mathrm{GG}+\mathrm{CG})$ vs. $\mathrm{CC}$ & $0.827(0.593-1.153)$ & 0.262 & $0.805(0.484-1.339)$ & 0.404 & $0.959(0.551-1.668)$ & 0.882 \\
\hline & recessive & GG vs. $(C G+C C)$ & $0.662(0.463-0.946)$ & 0.023 & $0.576(0.342-0.967)$ & 0.037 & $0.739(0.389-1.404)$ & 0.355 \\
\hline rs3766741 & dominant & $(\mathrm{GG}+\mathrm{CG})$ vs. $\mathrm{CC}$ & $0.639(0.433-0.943)$ & 0.024 & $0.605(0.334-1.096)$ & 0.098 & $0.665(0.345-1.282)$ & 0.224 \\
\hline
\end{tabular}

$\mathrm{OR}$, odds ratio; $\mathrm{CI}$, confidence interval; $\mathrm{SNP}$, single nucleotide polymorphism

OR a adjusted for gender, age, body mass index, total cholesterol, triglyceride, fasting glucose, smoking habits and drinking habits.

$\mathrm{OR}^{\mathrm{b}}$ adjusted for age, body mass index, total cholesterol, triglyceride, high-density lipoprotein, fasting glucose, smoking habits and drinking habits.

$\mathrm{OR}^{\mathrm{c}}$ adjusted for age, body mass index, total cholesterol, triglyceride, fasting glucose, smoking habits and drinking habits.

Table 4. Haplotype Analyses of the Mfn2 Polymorphisms in Hypertensive Cases and Control Subjects

\begin{tabular}{|c|c|c|c|c|c|c|c|c|c|c|c|}
\hline \multicolumn{6}{|c|}{ Haplotype } & \multicolumn{2}{|c|}{ Frequency } & \multirow[t]{2}{*}{$\mathrm{OR}^{\mathrm{a}}$} & \multirow[t]{2}{*}{$\mathrm{Pa}$} & \multirow[t]{2}{*}{$\mathrm{OR}^{\mathrm{b}}$} & \multirow[t]{2}{*}{$\mathrm{Pb}$} \\
\hline M1 & M2 & M3 & M4 & M5 & M6 & Case & Control & & & & \\
\hline $\mathrm{C}$ & $\mathrm{C}$ & $\mathrm{A}$ & A & $\mathrm{C}$ & $\mathrm{C}$ & 0.448 & 0.412 & 1.156 & 0.075 & 1.24 & 0.047 \\
\hline A & $\mathrm{T}$ & A & G & G & $\mathrm{C}$ & 0.365 & 0.393 & 0.886 & 0.145 & 0.641 & 0.423 \\
\hline A & $\mathrm{C}$ & A & G & G & G & 0.075 & 0.097 & 0.759 & 0.058 & 0.642 & 0.024 \\
\hline A & $\mathrm{C}$ & G & G & C & $\mathrm{C}$ & 0.06 & 0.057 & 1.056 & 0.754 & 0.971 & 0.893 \\
\hline C & C & G & G & $\mathrm{C}$ & $\mathrm{C}$ & 0.049 & 0.037 & 1.357 & 0.13 & 1.15 & 0.602 \\
\hline
\end{tabular}

M1: rs2336384, M2: rs2295281, M3:rs17037564, M4: rs2236057, M5: rs2236058, M6: rs3766741.

a ORs and P-values for the haplotype-based association analysis derived from comparing of a specific haplotype with the others.

bORs and P-values for the haplotype-based logistic regression analysis after adjusting for gender, age, body mass index, total cholesterol, triglyceride, fasting glucose,

smoking habits and drinking habits.

\section{Association analyses}

Logistic regression analyses were performed under different genetic models (dominant, recessive, additive) after adjusting for confounding variables including gender, age, BMI, TCHO, TG, glucose and the ratios of smoking and drinking habits. The results of logistic regression analyses were shown in Table 3. It showed that rs2336384 was significantly associated with $\mathrm{EH}$ risk under both the additive genetic model (CC vs. CA vs. AA: $\mathrm{P}=0.031, \mathrm{OR}=1.273$, 95\% CI $=1.023-1.584)$ and dominant genetic model $((\mathrm{CC}+\mathrm{CA}) \quad$ vs. $\mathrm{AA}: \mathrm{P}=0.005, \quad \mathrm{OR}=1.617$, 95\% CI $=1.155-2.264)$, which indicated that $C$ allele carriers of rs2336384 have a higher risk for EH. For rs2236058 polymorphism, significant association could be found in the additive genetic model (GG vs. CG. Vs. CC: $\mathrm{P}=0.041, \mathrm{OR}=0.802,95 \% \mathrm{CI}=0.649-0.991$ ) and in the recessive genetic model (GG vs. $(\mathrm{CG}+\mathrm{CC})$ : $\mathrm{P}=0.023$, OR $=0.662,95 \% \mathrm{CI}=0.463-0.946)$, which suggested that individuals carrying GG genotype of rs2236058 have a lower risk for EH. Furthermore, SNPs 2236057 and rs3766741 were significantly associated with $\mathrm{EH}$ under the dominant genetic models (rs2236057 (AA+AG) vs. GG: $\mathrm{P}=0.031, \mathrm{OR}=1.418$, 95\%CI=1.033-1.948; rs3766741 (GG+CG) vs. CC: $\mathrm{P}=0.024, \mathrm{OR}=0.639,95 \% \mathrm{CI}=0.433-0.943$, respectively). No significant association was found between rs2295281 or rs17037564 polymorphisms and EH risk. Gender-based subgroup analyses showed that significant association between rs2336384, rs2236057 and rs2236058 and EH could be found in males, but not in females. As for rs2295281, rs17037564 or rs3766741 polymorphisms, no significant association with $\mathrm{EH}$ were found either in males or in females.

\section{Haplotype analyses}

As shown in Figure 1, the haploview program revealed that the six polymorphisms of $M f n 2$ gene were in one linkage disequilibrium block and in linkage with each other. The haplotype analyses of the six polymorphisms of the $M f n 2$ gene in hypertensive patients and control subjects are shown in Table 4. By using the Haploview software, we found that the frequency of the haplotype CCAACC (rs2336384-rs2295281-rs17037564-rs2236057-rs2236058 -rs3766741) was obviously higher in the hypertensive cases $(44.8 \%)$ than in the normotensive controls 
$(41.2 \%)$, but it did not reach statistical significance $(p=0.075)$. After adjustment for the confounding variables (gender, age, BMI, TCHO, Glu, smoking and drinking habits), we found that the haplotype CCAACC was significantly associated with increased risk for $\mathrm{EH}(\mathrm{p}=0.047, \mathrm{OR}=1.156)$ with the PLINK software. In addition, there was a significant association between the haplotype ACAGGG and decreased risk for EH. No significant association was observed between the other haplotypes and $\mathrm{EH}$ risk.

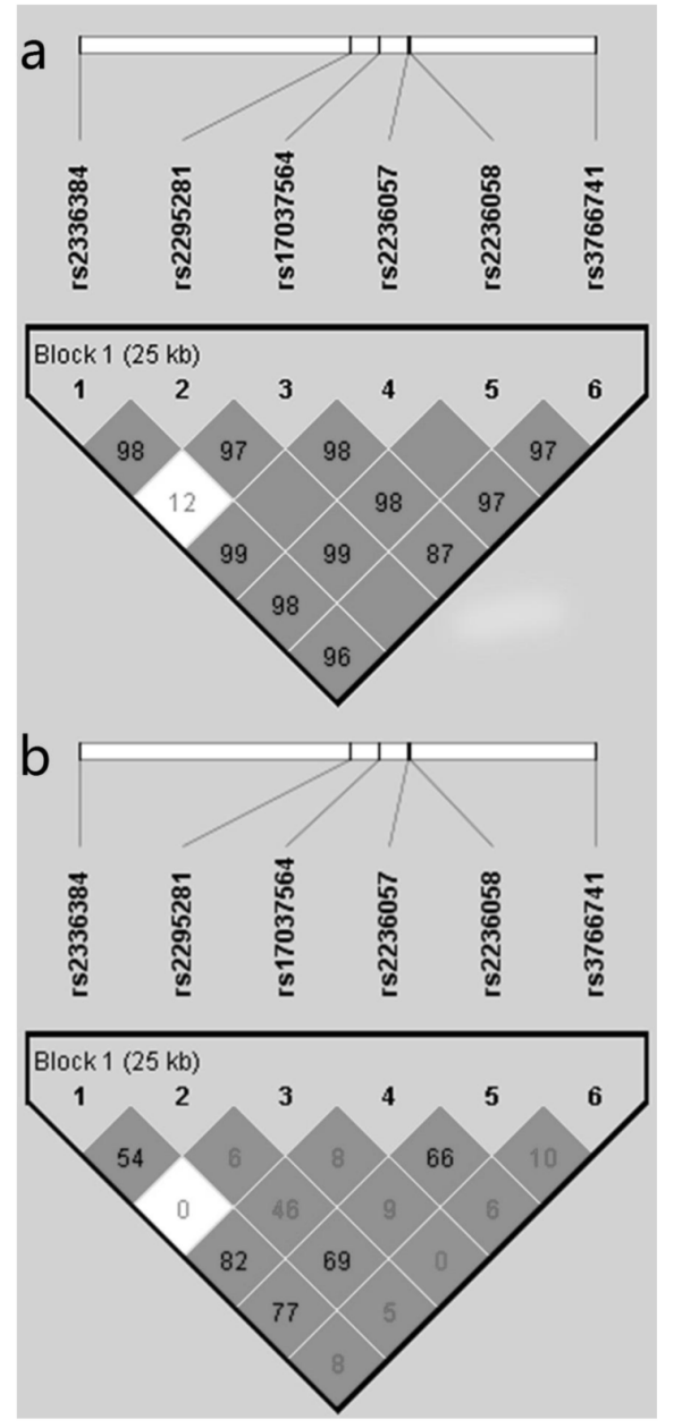

Figure 1. Linkage disequilibrium (LD) block defined by the Haploview program based on the solid spine of LD method. a. represent LD result of D'; b. represents LD result of $r^{2}$.

\section{Discussion}

In the present study, six tagging SNPs of the Mfn 2 gene were identified by Haploview software and genotyping was further performed. Multivariate logistic regression analyses were performed to ex- clude the influences of those confounding factors. The results showed that the rs2236384, rs2236057, rs2236058 and rs3766741 polymorphisms in the Mfn2 gene were significantly related to $\mathrm{EH}$ risk in the Northern Han Chinese population, and the haplotypes CCAACC and ACAGGG might be a protective factor and a risk factor, respectively. Subgroup analysis by gender showed that rs2236384, rs2236057 and rs2236058 polymorphisms were associated with EH risk in males, but not in females.

To our knowledge, there were three studies explored the relationship between gene Mfn2 polymorphisms and EH. In 2011, Wang et al. [17] selected seven candidate SNPs in intron 2 and investigated the association between these SNPs and EH and found that rs873457, rs2336384, rs1474868, rs4846085 and rs2236055 were significantly associated with $\mathrm{EH}$ in the Chinese. In 2013, Wang et al. [22] focused on the SNPs of 5'-uncoding region (UTR) of $M f n 2$ and explored the association between $-1248 \mathrm{~A}>\mathrm{G}$ variation of $\mathrm{M} f \mathrm{n} 2$ gene and hypertension in the Chinese. They found that 5'-UTR -1248 A>G variation of the Mfn2 gene was associated with hypertension in Chinese. The results of these studies indicated that $M f n 2$ gene polymorphisms played important roles in the development of hypertension. Considering that those SNPs included in Wang et al.'s studies could not represent all common SNPs and some southern Han Chinese subjects and central Han Chinese subjects were also enrolled, we selected tagging SNPs that capture most common SNPs in Mfn2 gene and performed another association research in a northern Han Chinese population. This population is characterized by genetic homogeneity and geographic stability and the participants are most likely uniform in their environmental exposures, including the habitual intake of high salt [23-25]. These characteristics are important in studying the genetics of essential hypertension. And we found that rs2336384 polymorphism was significantly associated with EH in northern Han Chinese, which is consistent with Wang et al.'s finding. In our study, rs2236057, rs2236058 and rs3766741 polymorphisms were also found to be significantly associated with EH. Although the selected SNPs were different in both studies, the results implied that the $M f n 2$ gene polymorphisms were associated with EH risk. In addition, our subgroup analysis by gender showed that these polymorphisms were male-specific, which was consistent with Wang et al.'s results. We considered that there are some reasons contributing to this phenomenon: 1) The genetic architecture of males and females are different [26], which indicates that EH susceptibility genes of males may differ from that of females; 2) The expression of $M f n 2$ gene can be regulated under different conditions including the exposure to 
cold, chronic exercise, and proinflammatory factors [27-29]. In China, male subjects have more risk factors of EH than females such as smoking, drinking, mental stress and less estrogen. We infer that these risk factors can interact with the genetic variation of $M f n 2$ influencing the regulation of blood pressure. However, Jin et al. [16]showed that no significant association between rs2336384 polymorphism and hypertension in Korean individuals, which was different from our finding. Given that the allele C frequency of rs2236384 was different ( $45.5 \%$ vs. $41.9 \%)$, we inferred that this inconsistence might be due to the different genetic background between Chinese and Korean. Studies in different populations are needed to confirm this finding.

As we know, a single SNP exerts a minor effect to one phenotype, but several SNPs tend to be linked tightly and influence the phenotype together. Therefore, the haplotype analysis has advantages over an analysis based on individual SNP for the genetic study of complex diseases such as EH [30]. In our study, the six SNPs were in close LD with each other and located in one block. Haplotype-based analysis showed that the haplotype CCAACC (rs2236384-rs2295281-rs17037564-rs22365057-rs223605 8-rs3766741) was significantly associated with an increased risk for EH after adjusting for the confounding variables, which was consistent with the findings of the association analysis between rs2236384 and rs2236057 and EH risk. These data imply that individuals in this population who harbor the haplotype CCAACC may be at risk to develop essential hypertension. In addition, we also found that the haplotype ACAGGG was significantly associated with a decreased risk for $\mathrm{EH}$, which was in consistence with the association analysis between rs2236058 and rs3766741 and $\mathrm{EH}$ risk. These data revealed that participants carrying the haplotype ACAGGG are less likely to develop essential hypertension. Differently, there was no haplotype-based analysis performed in the study of Jin et al. To some extent, our study provide more information about the gene $M f n 2$ and $\mathrm{EH}$.

Currently, there are several experimental researches about gene Mfn2, which may provide some support to the association between Mfn2 and EH. The expression of $M f n 2$ gene was reduced in hyper-proliferative VSMCs from SHR arteries, as well as in white blood cells, explanted-vessels and cultured VSMCs from hypertensive patients [7, 31]. Overexpression of Mfn2 overtly suppressed serum-evoked VSMC proliferation in vitro and this anti-proliferative effect was mediated by inhibiting extracellular signal-regulated kinase/mitogen-activated (ERK/ MAPK) signaling and subsequent cell-cycle arrest [7-9]. Earlier studies demonstrated that vascular hy- pertrophy is a major contributor to the elevated blood pressure in established genetic and experimental hypertension [32, 33]. In SHR, an increase in both the number and size of VSMCs has been reported to be responsible for the vascular hypertrophy [12, 13]. Based on the above information, we inferred Mfn2 may be involved in the pathogenesis of $\mathrm{EH}$ through negative modulating of VSMC proliferation. Besides the anti-proliferative effect, some studies reported that Mfn2 expression was reduced in skeletal muscle of obese subjects and in type 2 diabetic patients [29, 34]. Moreover, experiments in vivo indicated Mfn2 deficiency could lead to insulin resistance through increasing $\mathrm{H}_{2} \mathrm{O}_{2}$ concentration and impairing insulin signaling in liver and muscle [10]. Considering the role of insulin resistance and compensatory hyperinsulinemia in hypertension [14], we speculated that Mfn2 may participate in the development of $\mathrm{EH}$ through this mechanism. In addition, Young et al. showed recently that endoplasmic reticulum (ER) stress, notably brain ER stress, played a key role in chronic hypertension [15]. Besides located on the outer mitochondrial membrane and regulated the mitochondrial fusion, Mfn2 was also present in the ER and regulated the ER shape [35]. And experiments in vitro showed that Genetic ablation of Mfn2 in mouse embryonic fibroblasts amplified ER stress and exacerbated ER stress-induced apoptosis [11], which suggested that loss of Mfn2 promoted endoplasmic reticulum stress. Given that Mfn2 was expressed in brain, we considered that Mfn2 may influence $\mathrm{EH}$ through ER of central nervous system.

The introns play an important role in the regulation of gene expression in eukaryotes. Introns can influence gene expression through the presence of transcriptional regulatory elements such as enhancers or alternative promoters [36]. It was reported that many genes with an intact promoters cannot be expressed in the absence of an intron [37]. In the present study, we found four SNPs in the introns of $M f n 2$ significantly associated with EH. It was possible that this might be associated with the dys-regulated expression of Mfn2. The four positive polymorphisms of the Mfn2 gene in our study are all located in intron region, which indicates that they could potentially affect Mfn2 function through transcription regulation. We utilized the ENCODE module of UCSC Genome Bioinformatics (http:/ / genome.ucsc.edu/) and F-SNP (http://compbio.cs.queensu.ca/F-SNP/) to predict the potential function of the four positive polymorphisms [38]. F-SNP prediction indicated that these polymorphisms influence transcriptional regulation in different degrees. UCSC prediction showed that rs2336384 was about 20bp away from the potential transcription factor binding site (OLF1) and located in 
the CpG island regions, which indicate that they could potentially affect Mfn2 function through transcription regulation. Another possibility was that these polymorphisms were in linkage disequilibrium with other functional polymorphisms. However, the specific mechanisms of these polymorphisms in the development of hypertension need to be researched in further studies.

The subjects in the present study were all enrolled from northern Han ethnic group to reduce population stratification on some level. In addition, we selected hypertensive patients with a relatively early onset, and control subjects without a family history of hypertension to avoid selection bias. However, some limitations must be considered. First, given that there were no clues implying that Mfn2 gene was correlated with some hypertension biomarkers, therefore, we did not examine the association between these polymorphisms and biomarkers. We intend to focus on the downstream regulators of this gene involved in the regulation of hypertension to see if we can found the potential association between Mfn2 gene and hypertension biomarkers. Second, although the clinical progress and prognosis conditions of these participants were followed up, the current data obtained are not enough to assess the association between these polymorphisms and these conditions, which limited our understanding of the role of Mfn2 gene polymorphisms in the development of hypertension. Third, six common tagging SNPs were examined in our study, whereas other functional SNPs including low frequency SNPs are still worthy of study. Beyond this, functional studies at the molecular level could help determine the mechanism by which these positive SNPs can influence the function of Mfn2 gene and development of essential hypertension.

In conclusion, the present study found that the common variants (rs2336384, rs2236057, rs2236058 and rs3766741) of Mfn2 gene and the related haplotype CCAACC or ACAGGG were significantly associated with $\mathrm{EH}$ in northern Han Chinese population. In subgroup analyses, the rs2336384, rs2236057 and rs2236058 of $M f n 2$ were found to be significantly associated with $\mathrm{EH}$ in males, but not in females. Further functional studies of $M f n 2$ in essential hypertension are needed to confirm this discovery. Studies with larger sample size are needed to confirm these results and should be testified in different populations worldwide.

\section{Acknowledgements}

We are grateful to Jiapeng Zhou (Shijiazhuang Epigene Biological Technology Co., Ltd, China) for his help in the statistical analysis of the manuscript. This work was supported by grants from Beijing Natural Science Foundation of China (7120001) and the National High-tech Research and Development Projects (863) (2008AA02Z441).

\section{Competing Interests}

The authors have declared that no competing interest exists.

\section{References}

1. Kearney PM, Whelton M, Reynolds K, et al. Global burden of hypertension: analysis of worldwide data. Lancet 2005; 365(9455):217-223.

2. Gu D, Reynolds K, Wu X, et al. Prevalence, awareness, treatment, and control of hypertension in china. Hypertension 2002; 40(6):920-927.

3. Levy D, DeStefano AL, Larson MG, et al. Evidence for a gene influencing blood pressure on chromosome 17. Genome scan linkage results for longitudinal blood pressure phenotypes in subjects from the framingham heart study. Hypertension 2000; 36(4):477-483.

4. Levy D, Ehret GB, Rice K, et al. Genome-wide association study of blood pressure and hypertension. Nat Genet 2009; 41(6):677-687.

5. Yang HC, Liang YJ, Chen JW, et al. Identification of IGF1, SLC4A4, WWOX, and SFMBT1 as hypertension susceptibility genes in Han Chinese with a genome-wide gene-based association study. PLoS One 2012; 7(3):e32907.

6. Lu X, Wang L, Lin X, et al. Genome-wide association study in Chinese identifies novel loci for blood pressure and hypertension. Hum Mol Genet 2015; 24(3):865-874.

7. Chen KH, Guo X, Ma D, et al. Dysregulation of HSG triggers vascular proliferative disorders. Nat Cell Biol 2004; 6(9):872-883.

8. Xia Y, Wu Y, He X, et al. Effects of mitofusin-2 gene on cell proliferation and chemotherapy sensitivity of MCF-7. J Huazhong Univ Sci Technolog Med Sci 2008; 28(2):185-189.

9. de Brito OM, Scorrano L. Mitofusin 2: a mitochondria-shaping protein with signaling roles beyond fusion. Antioxid Redox Signal 2008; 10(3):621-633.

10. Sebastian D, Hernandez-Alvarez MI, Segales J, et al. Mitofusin 2 (Mfn2) links mitochondrial and endoplasmic reticulum function with insulin signaling and is essential for normal glucose homeostasis. Proc Natl Acad Sci U S A 2012; 109(14):5523-5528.

11. Ngoh GA, Papanicolaou KN, Walsh K. Loss of mitofusin 2 promotes endoplasmic reticulum stress. J Biol Chem 2012; 287(24):20321-20332.

12. Mulvany MJ, Baandrup U, Gundersen HJ. Evidence for hyperplasia in mesenteric resistance vessels of spontaneously hypertensive rats using a three-dimensional disector. Circ Res 1985; 57(5):794-800.

13. Owens GK, Schwartz SM. Alterations in vascular smooth muscle mass in the spontaneously hypertensive rat. Role of cellular hypertrophy, hyperploidy, and hyperplasia. Circ Res 1982; 51(3):280-289.

14. Reaven GM. Insulin resistance and compensatory hyperinsulinemia: role in hypertension, dyslipidemia, and coronary heart disease. Am Heart J 1991; 121(4 Pt 2):1283-1288.

15. Young CN, Cao X, Guruju MR, et al. ER stress in the brain subfornical organ mediates angiotensin-dependent hypertension. J Clin Invest 2012; 122(11):3960-3964.

16. Jin HS, Sober S, Hong KW, et al. Age-dependent association of the polymorphisms in the mitochondria-shaping gene, OPA1, with blood pressure and hypertension in Korean population. Am J Hypertens 2011; 24(10):1127-1135.

17. Wang Z, Liu Y, Liu J, et al. HSG/Mfn2 gene polymorphism and essential hypertension: a case-control association study in Chinese. J Atheroscler Thromb 2011; 18(1):24-31.

18. Gu D, Su S, Ge D, et al. Association study with 33 single-nucleotide polymorphisms in 11 candidate genes for hypertension in Chinese. Hypertension 2006; 47(6):1147-1154.

19. Ge D, Huang J, He J, et al. beta2-Adrenergic receptor gene variations associated with stage-2 hypertension in northern Han Chinese. Ann Hum Genet 2005; 69(Pt 1):36-44.

20. Li Y, Gu S, Wu O, et al. No association of ERCC1 C8092A and T19007C polymorphisms to cancer risk: a meta-analysis. Eur J Hum Genet 2007; 15(9):967-973.

21. Martinez CM, Ertel A, Deloach S, et al. Variants in genes involved in functional pathways associated with hypertension in African Americans. Clin Transl Sci 2010; 3(6):279-286.

22. Wang Z, Liu $Y$, Liu J, et al. A novel 5'-uncoding region -1248 A>G variation of mitofusin-2 gene is associated with hypertension in Chinese. Yonsei Med J 2013; 54(3):603-608.

23. Xu S, Yin X, Li S, et al. Genomic dissection of population substructure of Han Chinese and its implication in association studies. Am J Hum Genet 2009; 85(6):762-774.

24. Wen B, Li H, Lu D, et al. Genetic evidence supports demic diffusion of Han culture. Nature 2004; 431(7006):302-305. 
25. Zhao L, Stamler J, Yan LL, et al. Blood pressure differences between northern and southern Chinese: role of dietary factors: the International Study on Macronutrients and Blood Pressure. Hypertension 2004; 43(6):1332-1337.

26. Ober C, Loisel DA, Gilad Y. Sex-specific genetic architecture of human disease. Nat Rev Genet 2008; 9(12):911-922.

27. Soriano FX, Liesa M, Bach D, et al. Evidence for a mitochondrial regulatory pathway defined by peroxisome proliferator-activated receptor-gamma coactivator-1 alpha, estrogen-related receptor-alpha, and mitofusin 2. Diabetes 2006; 55(6):1783-1791.

28. Fealy CE, Mulya A, Lai N, et al. Exercise training decreases activation of the mitochondrial fission protein dynamin-related protein-1 in insulin-resistant human skeletal muscle. J Appl Physiol (1985) 2014; 117(3):239-245.

29. Bach D, Naon D, Pich S, et al. Expression of Mfn2, the Charcot-Marie-Tooth neuropathy type 2A gene, in human skeletal muscle: effects of type 2 diabetes, obesity, weight loss, and the regulatory role of tumor necrosis factor alpha and interleukin-6. Diabetes 2005; 54(9):2685-2693.

30. Morris RW, Kaplan NL. On the advantage of haplotype analysis in the presence of multiple disease susceptibility alleles. Genet Epidemiol 2002; 23(3):221-233.

31. Liu YP, Wen SJ, Liu Y, et al. [Hyperplasia suppressor gene expression in vascular smooth muscle cells derived from normotensive and hypertensive patients underwent bypass surgery]. Zhonghua Xin Xue Guan Bing Za Zhi 2007; 35(10):914-918.

32. Folkow B. Physiological aspects of primary hypertension. Physiol Rev 1982; 62(2):347-504.

33. Korner PI, Bobik A, Angus JA, et al. Resistance control in hypertension. J Hypertens Suppl 1989; 7(4):S125-S134, S135.

34. Hernandez-Alvarez MI, Thabit $\mathrm{H}$, Burns N, et al. Subjects with early-onset type 2 diabetes show defective activation of the skeletal muscle PGC-1\{alpha\}/Mitofusin-2 regulatory pathway in response to physical activity. Diabetes Care 2010; 33(3):645-651.

35. de Brito OM, Scorrano L. Mitofusin 2 tethers endoplasmic reticulum to mitochondria. Nature 2008; 456(7222):605-610.

36. Barrett LW, Fletcher S, Wilton SD. Regulation of eukaryotic gene expression by the untranslated gene regions and other non-coding elements. Cell Mol Life Sci 2012; 69(21):3613-3634.

37. Rose $\mathrm{AB}$. Intron-mediated regulation of gene expression. Curr Top Microbiol Immunol 2008; 326:277-290.

38. Lee PH, Shatkay H. F-SNP: computationally predicted functional SNPs for disease association studies. Nucleic Acids Res 2008; 36(Database issue):D820-D824 